\title{
Social Stress Enhances Sympathetic Innervation of Primate Lymph Nodes: Mechanisms and Implications for Viral Pathogenesis
}

\author{
Erica K. Sloan, ${ }^{1}$ John P. Capitanio, ${ }^{3,4}$ Ross P. Tarara, ${ }^{3}$ Sally P. Mendoza, ${ }^{3}$ William A. Mason, ${ }^{3}$ and Steve W. Cole ${ }^{1,2}$ \\ ${ }^{1}$ Department of Medicine, Division of Hematology-Oncology, University of California Los Angeles (UCLA) School of Medicine, UCLA AIDS Institute, \\ Cousins Center for Psychoneuroimmunology at the Semel Institute of Neuroscience and Human Behavior, ${ }^{2} J o n s s o n$ Comprehensive Cancer Center and \\ UCLA Molecular Biology Institute, Los Angeles, California 90095, and ${ }^{3}$ California National Primate Research Center and ${ }^{4}$ Department of Psychology, \\ University of California Davis, Davis, California 95616
}

Behavioral processes regulate immune system function in part via direct sympathetic innervation of lymphoid organs, but little is known about the factors that regulate the architecture of neural fibers in lymphoid tissues. In the present study, we find that experimentally imposed social stress can enhance the density of catecholaminergic neural fibers within axillary lymph nodes from adult rhesus macaques. This effect is linked to increased transcription of the key sympathetic neurotrophin nerve growth factor and occurs predominately in extrafollicular regions of the paracortex that contain T-lymphocytes and macrophages. Functional consequences of stressinduced increases in innervation density include reduced type I interferon response to viral infection and increased replication of the simian immunodeficiency virus. These data reveal a surprising degree of behaviorally induced plasticity in the structure of lymphoid innervation and define a novel pathway by which social factors can modulate immune response and viral pathogenesis.

Key words: stress; sympathetic nervous system; plasticity; NGF; interferon; SIV

\section{Introduction}

Neuroanatomical studies have shown that all primary and secondary lymphoid organs receive direct innervation from the sympathetic division of the autonomic nervous system (Madden et al., 1995a; Bellinger et al., 2001). Postganglionic fibers from the sympathetic nervous system (SNS) enter lymphoid organs in association with the vasculature and subsequently radiate into parenchymal tissues containing lymphocytes and antigenpresenting cells (Bellinger et al., 2001). Structural varicosities situated periodically over the length of these fibers release micromolar concentrations of the catecholamine neurotransmitter norepinephrine (NE) in response to stress and other stimuli (Shimizu et al., 1994; Madden et al., 1995a). NE signals leukocytes via cellular $\beta$-adrenergic receptors, which activate the cAMP/PKA (protein kinase A) signaling cascade to regulate a

\footnotetext{
Received Aug. 24, 2006; revised June 26, 2007; accepted July 2, 2007.

This work was supported by National Institute of Mental Health Grant MH049033, National Institutes of Allergy and Infectious Disease and Neurological Disorders and Stroke Grant AI/NS052737, University of California Universitywide AIDS Research Program Grant CC99-LA-02, the Norman Cousins (enter at University of California Los Angeles (UCLA), the UCLA AIDS Institute, and the James B. Pendelton Charitable Trust. We thank Suzanne Stevens and Srinivasan ThyagaRajan for glyoxylic acid chemofluorescence protocols; Ding Lu and the Immunology Core Laboratory at California National Primate Research Center (CNPRC) for SIV ${ }_{\text {mac251 }}$ stock and SIV culture protocols; Harry Vinters and the UCLA AIDS Institute for access to a BSL2+ cryostat; Christine Brennan, Erna Tarara, Greg Vicinio, Carmel Stanko, Laura Del Rosso, Kristen Cooman, and the Veterinary, Animal Care, and Research Services staff of CNPRC for study conduct; Christina Nguyen and Benjamin Cox for assistance with data analysis; and Jerome Zack, John Cacioppo, John Sheridan, James Bisley, and Michael Irwin for their thoughtful discussions of this research.

Correspondence should be addressed to Steve W. Cole, Department of Medicine, Division of Hematology-Oncology, 11-934 Factor Building, David Geffen School of Medicine at University of California Los Angeles, Los Angeles, CA 90095-1678. E-mail: coles@ucla.edu.

DOI:10.1523/JNEUROSCI.1247-07.2007

Copyright $\odot 2007$ Society for Neuroscience $\quad$ 0270-6474/07/278857-09\$15.00/0
}

variety of immune processes including leukocyte activation, cytokine production, and cell trafficking (Kammer, 1988; Ottaway and Husband, 1994; Madden et al., 1995a; Cole et al., 1998, 1999; Sanders and Straub, 2002). Pharmacologic blockade of SNS activity can alter in vivo immune responses to model antigens and pathogen challenges (Madden et al., 1994; Kohm and Sanders, 1999). Effects of physiological variations in SNS activity are less well understood, but direct sympathetic innervation of lymphoid tissue is believed to constitute one major pathway by which behavioral processes can affect immune function (Felten et al., 1987; Madden et al., 1994, 1995b; Bellinger et al., 2001).

Most research on lymphoid innervation has presumed that acute changes in neural activity constitute the primary mechanism by which behavioral factors alter NE levels within lymphoid organs (Shimizu et al., 1994). However, a small number of studies have documented structural changes in the pattern of lymphoid innervation with aging and inflammation (Madden et al., 1997; Kelley et al., 2003; Sloan et al., 2006). Those results suggest an alternative model in which long-term changes in innervation density might regulate lymph node biology independently of short-term variations in neural activity. No studies have documented a behavioral influence on the structure of lymphoid innervation, but one recent analysis found that physical (auditory) stress could alter peptidergic innervation of the skin within a surprisingly short period of time $(\sim 48 \mathrm{~h})$ (Peters et al., 2005).

In the present study, we sought to determine whether chronic social stress might alter the density of catecholaminergic innervation in secondary lymphoid tissue and, if so, what molecular mechanisms might contribute to those dynamics. Given the key 
role that secondary lymphoid organs play in initiating immune responses, we also sought to determine how stress-induced changes in lymphoid innervation might impact the immune response to a viral infection.

\section{Materials and Methods}

Social stress. Thirty-six male rhesus macaques aged 7-10 years were relocated from outdoor social groups to individual housing where they lived throughout the experiment. Animals were given social experience $4 \mathrm{~d}$ per week for $100 \mathrm{~min}$ per day over 39 weeks, in cages measuring $1.8 \times 3.1 \times$ $2.2 \mathrm{~m}$. Each animal was randomly assigned to either the stable social condition (the same three animals met daily) or the unstable social condition (two to four animals per group, with number and identity of partners changing on a daily basis) (Capitanio et al., 1998). Unstable social conditions prompt continual reestablishment of social dominance hierarchies, resulting in behavioral and neuroendocrine indications of stress (e.g., threat behavior and altered cortisol response) (Capitanio et al., 1998). Twenty-four animals were infected with simian immunodeficiency virus (SIV) during the second week of the social manipulation period. Primary analyses focus on uninfected animals, with SIV infection serving as a challenge model to evaluate functional changes in immune response.

SIV infection. Randomly assigned animals were inoculated with $10^{2.66}$ $50 \%$ tissue culture infective dose of $\mathrm{SIV}_{\text {mac251 }}$, and all inoculated animals became infected as documented by reverse transcription (RT)-PCR detection of plasma viral RNA (Sloan et al., 2006) and development of SIV-specific antibodies (data not shown). Progression of SIV pathogenesis was assessed 36 weeks after inoculation by branched DNA assay of plasma SIV viral load (Chiron, Emeryville, CA) and flow cytometric quantification of peripheral blood CD4 + T-lymphocyte levels $(\mathrm{CD} 3+$ / $\mathrm{CD} 4+$ staining in lymphocytes defined by forward- versus side-scatter gating on a FACSCalibur cytometer) (BD Biosciences, San Jose, CA). As key sites of SIV replication in vivo (Fox et al., 1991), lymph nodes were biopsied 37 weeks after inoculation, during the chronic stage of infection ( $>6$ months after establishment of viral replication set point, and at least 2 months before onset of clinical disease, except for one animal with SIV mortality $9 \mathrm{~d}$ after lymph node biopsy). Animals were healthy at the time of biopsy, and lymph nodes were generally unreactive with minimal signs of hyperplasia (Sloan et al., 2006). Assessment of plasma viral load and in situ hybridization of SIV replication sites within lymph node sections were performed as previously described (Sloan et al., 2006).

In vitro analyses of SIV replication used adult male rhesus macaque peripheral blood mononuclear cells (PBMCs) that were infected with $\mathrm{SIV}_{\text {mac251 }}$, stimulated with phytohemagglutinin (PHA) (Sigma, St. Louis, $\mathrm{MO}$ ), and subsequently cultured in the presence of $0.5,5$, or 50 $\mathrm{ng} / \mathrm{ml}$ biologically active recombinant human mature NGF $\beta$ (US Biological, Swampscott, MA) or saline control. Viral replication at 4 and $6 \mathrm{~d}$ after infection was quantified by real-time RT-PCR (see below for description) for gag and env mRNA in total cellular RNA using previously published primer sequences (Canto-Nogues et al., 2001). Results were normalized to expression of cellular mRNA for $\beta$-actin (ACTB; primer sequences below).

Lymph node innervation. Four axillary lymph nodes were biopsied from each of 20 macaques after 39 weeks of stable versus unstable social conditions (including $11 \mathrm{SIV}+$ animals that remained alive 37 weeks after virus inoculation). One to three lymph nodes from each of seven uninfected macaques (14 nodes) and nine SIV-infected macaques (12 nodes) were randomly selected for histological analysis. The distribution of catecholaminergic varicosities was mapped in $16 \mu \mathrm{m}$ cryostat sections using glyoxylic acid chemofluorescence as described previously (Sloan et al., 2006). NE is believed to be released from structural varicosities situated periodically along the length of catecholaminergic fibers (Bellinger et al., 2001). To estimate the density of functional innervation, we used the stereological approach described by Mouton (2002): (1) lymph nodes were sectioned in random orientation to provide a random twodimensional sample through three-dimensional space, (2) a grid of $250 \times 250 \mu \mathrm{m}$ spatial units (quadrates) was superimposed as a reference frame to allow counting of catecholaminergic varicosities across the en- tire section (see Fig. 1c,d), and (3) the Delesse area-volume principle was applied to estimate the three-dimensional density of catecholaminergic varicosities from two-dimensional varicosity frequency data. By satisfying each of these criteria, this approach gives an unbiased estimate of relative three-dimensional innervation density from a two-dimensional histological profile (Mouton, 2002). Tissue shrinkage may occur during the freezing process and could occur unevenly across a tissue section, but this dynamic would not differentially affect tissues from differing experimental conditions. Thus, tissue shrinkage would not bias measures of relative innervation density used in this study. Innervation density was estimated at the level of the whole lymph node, and within functionally distinct anatomical regions (e.g., paracortex, cortex, and medulla). Anatomical subregions were mapped on adjacent hematoxylin and eosin (H\&E)-stained section by a veterinary pathologist (R. P. Tarara) blinded to localization of catecholaminergic fibers. Tissue sections were gridded into $250 \mu \mathrm{m}^{2}$ quadrates and each quadrate was assigned to a distinct anatomical subregion based on H\&E staining. Quadrates that spanned two or more subregions were divided into eighths, and each eighth was allocated to a specific subregion based on $\mathrm{H} \& \mathrm{E}$ anatomical information. By allowing each varicosity to be counted only once, this process ensured that density estimates were unbiased within distinct subregions and statistically independent across subregions. All assays were performed blind to information on social conditions.

Expression of neurotrophic factors and cytokines. Real-time RT-PCR was used to quantify NGF, IFNA, IFNB, IFNG, LIF, and IFI27 (ISG12) mRNA isolated from $3 \mathrm{mg}$ of lymph node tissue or from $10^{6}$ rhesus PBMCs treated with $10^{-6}$ M NE (Sigma) for 4, 8, 12, or $24 \mathrm{~h}$. Total RNA was isolated using RNeasy (Qiagen, Valencia CA), and cDNA was synthesized by iScript reverse transcriptase according to the manufacturer's protocol (Bio-Rad, Hercules, CA). Real-time PCR used iQ SYBR Green Supermix (Bio-Rad) with 45 PCR amplification cycles of $15 \mathrm{~s}$ of strand separation at $95^{\circ} \mathrm{C}$ and $60 \mathrm{~s}$ of annealing and extension at $60^{\circ} \mathrm{C}$. Triplicate determinations were quantified by threshold cycle analysis of SYBR Green fluorescence intensity using iCycler software (Bio-Rad), and normalization to parallel-amplified GAPDH and ACTB mRNA (Collado-Hidalgo et al., 2006). Primer sequences and gene regions that were amplified include the following: NGF, GenBank XM_001100522 (bp 434-601): forward (F), 5' GTTTTACCAAGGGAGCAGCTTTC-3', and reverse (R), 5'-TAGTCCAGTGGGCTTGGGGGA-3'; IFNA (conserved 368 bp sequence of 23 IFNA family members): F, 5' -AGAATCTCTCCTTTCTCCTG-3', and R, 5'-TCTGACAACCTCCCAGGCACA-3'; IFNB, GenBank EF064725 (bp 2263-2358): F, 5'-AAGGAGGACGCCGCATT-3', and R, 5'-AATAGTCTCATTCCAGCCAGTGC-3'; IFNG, GenBank A4376145 (bp 346-423): F, 5'-GAAAAGCTGACCAATTATTCGGTAA-3', and R, 5' AGCCATCACTTGGATGAGTTCA-3'; LIF, GenBank BC093733 (bp 222-297): F, 5'-TGCCAATGCCCTCTTTATTC-3', and R, 5'-GCCACATAGCTTGTCCAGGT-3'; IFI27, GenBank BC015492 (bp 456-553): F, 5'-TCACTGGGAGCAACTGGACTC-3', and R, 5'-GGGAGCTAGTAGAACCTCGCAAT-3'; GAPDH, GenBank BC083511 (bp 58-226): F, 5' GAAGGTGAAGGTCGGAGTC-3', and R, 5' -GAAGATGGTGATGGGATTC-3'; ACTB, GenBank NM_001101 (bp 549-573): F, 5'TCACCCACACTGTGCCCATCTACGA-3', and R, 5'-CAGCGGAACCGCTCATTGCCAATGG-3'.

NGFR and NTRK1 mRNA were amplified using TaqMan Gene Expression Assays (Hs00176787_m1 and Hs00609976_m1, respectively) (Applied Biosystems, Foster City, CA) using Quantitect Probe RT-PCR kit (Qiagen) and the cycling conditions described above.

Detection of NGF protein. Lymph nodes from healthy uninfected animals were homogenized in lysis buffer (10 mM Tris, pH 7.4, 1\% SDS) and proteins were separated by SDS-PAGE on a $15 \%$ polyacrylamide gel and transferred to nitrocellulose membrane (Hybond; Amersham Biosciences, Piscataway, NJ). Membranes were incubated with a rabbit antiNGF polyclonal antibody $(\mathrm{H}-20 ; 1 \mu \mathrm{g} / \mathrm{ml}$; Santa Cruz Biotechnology, Santa Cruz, CA) overnight at $4^{\circ} \mathrm{C}$, followed by incubation with horseradish peroxidase-conjugated secondary antibody and detection with ECL Plus (Amersham Biosciences). Similar results were obtained by immunoprecipitation with rabbit anti-NGF serum (Cedarlane, Burlington, $\mathrm{NC}$ ) in $50 \mathrm{~mm}$ Tris- $\mathrm{HCl}, \mathrm{pH} 8.0,0.5 \mathrm{~mm}$ EDTA, $125 \mathrm{~mm} \mathrm{NaCl}, 0.5 \%$ 
NP-40, $10 \%$ glycerol, $0.1 \%$ SDS, complete Mini EDTA-free protease inhibitors (Roche Diagnostics, Indianapolis, IN) (data not shown).

Activation of TrkA receptors in situ. Active TrkA was localized by immunostaining of lymph node tissue sections with polyclonal rabbit antibody to phosphorylated TrkA ( $10 \mu \mathrm{g} / \mathrm{ml}$; Upstate, Waltham, MA) using the method detailed below (see below, Leukocyte distribution in situ). Adjacent sections were stained with glyoxylic acid chemofluorescence (see above, Lymph node innervation) to confirm catecholaminergic neural fibers.

Leukocyte distribution in situ. Frozen lymph node sections were postfixed in $4 \%$ paraformaldehyde and permeabilized with $0.2 \%$ Triton X-100/2\% normal goat serum (Dako North America, Carpinteria, CA) in PBS. Sections were incubated with primary antibodies or appropriate control antibodies in blocking solution [0.1 M Tris, pH 7.4, $0.15 \mathrm{M} \mathrm{NaCl}$, $2 \%$ normal goat serum, 2\% normal horse serum (Hyclone, Logan, UT)] at $4^{\circ} \mathrm{C}$ overnight, followed by secondary antibody (and tertiary detection as described below) at room temperature for $1 \mathrm{~h}$. Nuclei were counterstained with $1 \mu \mathrm{g} / \mathrm{ml}$ Hoechst 33342 (Sigma) for $1 \mathrm{~min}$ and sections were coverslipped with fluorescent mounting medium (Dako North America). Antibodies used were CD3 rabbit polyclonal antibody $(6.0 \mu \mathrm{g} / \mathrm{ml}$; DakoCytomation) followed by Alexa 488-conjugated goat anti-rabbit $\mathrm{F}\left(\mathrm{ab}^{\prime}\right)_{2}$ fragments (Invitrogen, Carlsbad, CA), CD20 clone L26 (0.45 $\mu \mathrm{g} / \mathrm{ml}$; Zymed, South San Francisco, CA) followed by Alexa 488conjugated goat anti-mouse $\mathrm{F}\left(\mathrm{ab}^{\prime}\right)_{2}$ fragments (Invitrogen), and macrophage marker HAM56 $(0.7 \mu \mathrm{g} / \mathrm{ml}$; Dako North America) or follicular dendritic cell marker CAN.42 (1.4 $\mu \mathrm{g} / \mathrm{ml}$; Dako North America) followed by biotinylated goat anti-mouse IgM $(2 \mu \mathrm{g} / \mathrm{ml}$; Vector Laboratories, Burlingame, CA) and tertiary Alexa 568-conjugated streptavidin (Invitrogen). All Alexa conjugates were used at $2 \mu \mathrm{g} / \mathrm{ml}$. For analysis of immunofluorescence coexpression, each field was imaged at $200 \times$ magnification by multichannel fluorescence microscopy for antibody staining of cell surface markers (CD3/HAM46 or CD20/FDC) and Hoechst 33342 nuclear stain, and single-channel images were overlaid using Photoshop 7.0 to assess colocalization. Images were acquired using an Axioskop 2 microscope, AxioCam HRc color camera, and AxioVision 4.1 software (Zeiss, Thornwood, NY). Relative frequency of T-lymphocytes was determined by averaging the pixel density of CD3 + staining in three microscope fields selected randomly from paracortical regions in randomly selected lymph nodes. Parallel analyses assessed the relative frequency of macrophages (HAM56+), B-lymphocytes (CD20+), and follicular dendritic cells (CAN.42+). Total cellular density was assayed by counting nuclei in five randomly selected $250 \mu \mathrm{m}^{2}$ quadrates from paracortex, cortex, and medulla in H\&E-stained sections photographed under brightfield. Total lymph node size and relative size of anatomical subcompartments were derived from total frequencies of $250 \mu \mathrm{m}^{2}$ quadrates in each lymph node (including fractional regions) (see above, Lymph node innervation) according to the Delesse area-volume principle (Mouton, 2002).

Statistical analysis. Spatial correlation analyses were performed as described previously (Sloan et al., 2006), with statistical mediation analyses (Hoyle and Kenny, 1999) conducted in the context of generalized linear models fit using SAS, version 9.1, PROC GLM (SAS Institute, Cary, NC). Analyses conducted at the level of individual tissue quadrates controlled for correlation among observations from the same tissue sample by treating each quadrate as a random observation nested within tissue sample, and each tissue sample as a random effect nested within experimental conditions (Miller, 1986). Simultaneous analysis of social influences and neuroanatomical influences on SIV gene expression modeled the density of active viral replication sites in tissue quadrate $i$ from animal $a\left(y_{a i}\right)$ as a function of (1) the number of catecholaminergic varicosities present in that quadrate $\left(\alpha_{a i}\right),(2)$ any generalized effect of social condition not mediated by proximity to sympathetic varicosities $\left(\beta_{c}=1\right.$ for all quadrates in tissues from animals socialized under unstable conditions vs 0 for quadrates in tissue from animals socialized under stable conditions), (3) any differential functional effect of varicosities under stable versus unstable conditions ( $\gamma=$ multiplicative product of $\alpha$ and $\beta$ ), and (4) individual differences across animals (nested within experimental condition; $\delta)$ (i.e., $y_{a i}=\alpha_{a i}+\beta_{c}+\gamma_{a i c}+\delta_{a}+\varepsilon_{a i}$, with $\varepsilon$ representing an independent random residual) (Miller, 1986).

\section{Results}

\section{Effects of social stress on behavior}

Animals were randomly assigned to 39 weeks of daily social interaction in either stable conditions (same animals met every day) or unstable conditions (group composition changed daily). Unstable social conditions have been shown to induce neuroendocrine and behavioral indications of stress in previous studies (Capitanio et al., 1998), and monitoring of agonistic social behavior confirmed those effects in the present sample. Relative to animals socialized in stable groups, those subjected to unstable social conditions showed a more than eightfold increase in the frequency of threat behaviors (mean \pm SE, $0.402 \pm 0.130$ per animal per week vs $0.047 \pm 0.014$ for controls; $p=0.041$ ), physical aggression $(0.034 \pm 0.011$ vs $0.000 \pm 0.000 ; p=0.028)$, and chases $(0.028 \pm 0.015$ vs $0.000 \pm 0.000 ; p=0.103)$.

\section{Effects of social stress on lymph node innervation}

To assess effects of behavioral stress on lymphoid innervation, we used glyoxylic acid chemofluorescence (de la Torre and Surgeon, 1976; Guidry, 1999; Sloan et al., 2006) to map the distribution of parenchymal and perivascular catecholaminergic neural fibers in 14 axillary lymph nodes sampled from adult male rhesus macaques (Fig. 1a,b). In lymph nodes biopsied from animals subject to unstable social conditions, stereological analysis revealed an $80 \%$ increase in the density of parenchymal catecholaminergic varicosities compared with tissues from animals socialized in stable conditions (Fig. $1 c-e$ ). Across 4582 individual $250 \mu \mathrm{m}^{2}$ lymph node tissue quadrates analyzed, the absolute density of parenchymal catecholaminergic varicosities increased from an average $1.32 \pm 0.13$ per quadrate under stable social conditions to $2.38 \pm 0.25$ under unstable social conditions (Fig. 1e) $(p<$ $0.0001)$. Stress-induced increases in the density of catecholaminergic varicosities occurred specifically within the lymph node parenchyma, and the density of perivascular varicosities did not change significantly $(p=0.332)$ (Fig. $1 e$ ).

Analyses performed within functionally distinct subregions of the lymph node showed that social stress enhanced lymphoid innervation specifically within the paracortex, a subregion populated primarily by T-lymphocytes and macrophages (Fig. $2 a-c$ ). Catecholaminergic varicosities were rarely found in the vicinity of B-lymphocytes (Fig. $2 d$ ). Social stress did not significantly alter the overall frequencies of T-lymphocytes, B-lymphocytes, macrophages, or follicular dendritic cells within lymph node tissue (all differences, $p>0.10$ ). Social conditions also had no detectable effect on the relative size of lymph nodes or their total cellular density (both $<10 \%$ difference; both $p>0.267$ ). All lymph nodes analyzed in this study showed fully structured medulla, paracortex, and cortex, with no effect of social conditions on the relative size of these subregions within the total lymph node area (all differences, $p>0.643$ ). Thus, social stress appears to focally remodel the sympathetic innervation of lymphoid tissue without significantly impacting the gross morphology or cellular composition of the organ.

\section{Neurotrophic factors}

To identify molecular mechanisms that might mediate the effects of social stress on lymphoid innervation, we surveyed the expression of genes known to modulate neural growth and maintenance. Tissues from socially stressed animals showed significantly higher concentrations of mRNA for NGF (mean 31\% increase over tissues from unstressed animals; $p=0.011$ ), which plays a key role in the development of the SNS (Levi-Montalcini, 
1987; Farinas, 1999). Parallel analyses of two cytokines known to retract sympathetic dendrites, interferon- $\gamma($ IFN- $\gamma)$ and leukemia inhibitory factor (LIF) (Guo et al., 1997; Kim et al., 2002), showed no stress-induced inhibition, but rather significant increases in both $(51 \%$ increase in LIF mRNA and $225 \%$ increase in IFNG; both $p<0.01$ ).

Consistent with the key role of NGF in supporting peripheral sympathetic innervation (Angeletti et al., 1971; Ruit et al., 1990; Tsui-Pierchala et al., 2002), levels of NGF mRNA showed a strong linear relationship to density of parenchymal catecholaminergic varicosities (Fig. 3a). However, tissue levels of NGF mRNA showed no relationship to the density of perivascular innervation (Fig. $3 b$ ). Neurotrophic activity is generally attributed to mature NGF (Edwards et al., 1988; Patel et al., 2000; Chao et al., 2006), although its pro-NGF precursor may also support neural survival (Lakshmanan et al., 1989; Chen et al., 1997; Fahnestock et al., 2004). Western blot analysis identified both mature NGF and pro-NGF protein within lymph node tissues (Fig. $3 c$ ). Consistent with NGF signaling through the TrkA-p75 receptor complex on neural fibers (Hempstead et al., 1991; Klein et al., 1991), immunofluorescence analyses identified phosphorylated TrkA (Fig. $3 d$ ) in catecholaminergic fibers within the lymph node parenchyma (Fig. 3e). Approximately $10 \%$ of catecholaminergic fibers stained positive for phosphorylated TrkA.

To determine whether stress-induced increases in NGF expression might be quantitatively sufficient to account for effects of social conditions on parenchymal innervation, we performed multivariate statistical analyses of mediation (Goodman, 1960; Hoyle and Kenny, 1999) (Fig. $3 f)$. Variations in NGF gene expression were sufficient to account for $86 \%$ of the total quantitative relationship between so-

cial stress and parenchymal varicosity density (mediational path, $p=0.004$; residual effect of stress, $p=0.321$ ) (Goodman, 1960; Hoyle and Kenny, 1999). Similar analyses of IFNG and LIF indicated that neither of those molecules was a plausible quantitative mediator of stress effects on lymphoid innervation (IFNG: mediational path, $p=0.109$; residual effect of stress, $p=0.038$; LIF: mediational path, $p=0.154$; residual effect of stress, $p=0.073$ ). Statistical nonsignificance of the later results, and previous data indicating that IFNG and LIF expression would be expected to inhibit sympathetic nerve fibers (Guo et al., 1997; Kim et al., 2002), are both consistent with the idea that these factors do not mediate stress-induced sympathetic innervation of lymphoid tissues.

The present data are consistent with the hypothesis that upregulation of NGF gene expression is responsible for increased innervation density in stressed animals, but a reverse relationship is conceivable in which catecholamine signaling might causally e

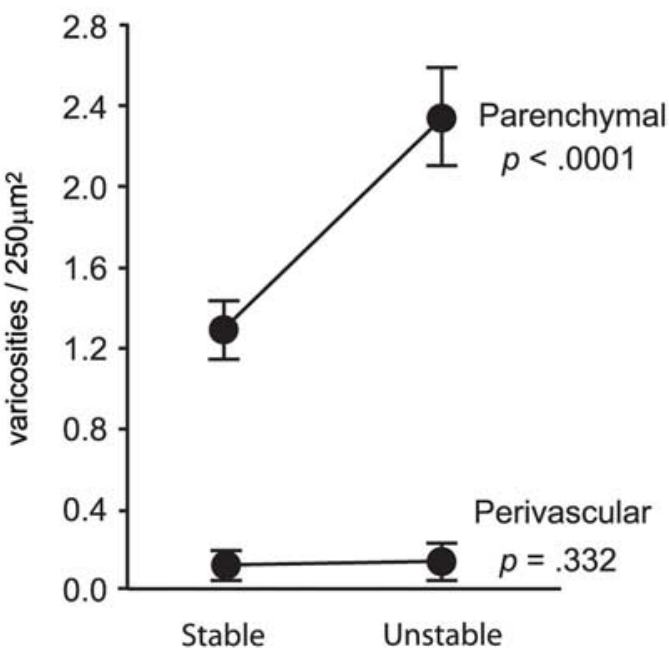

d

Unstable social grouping

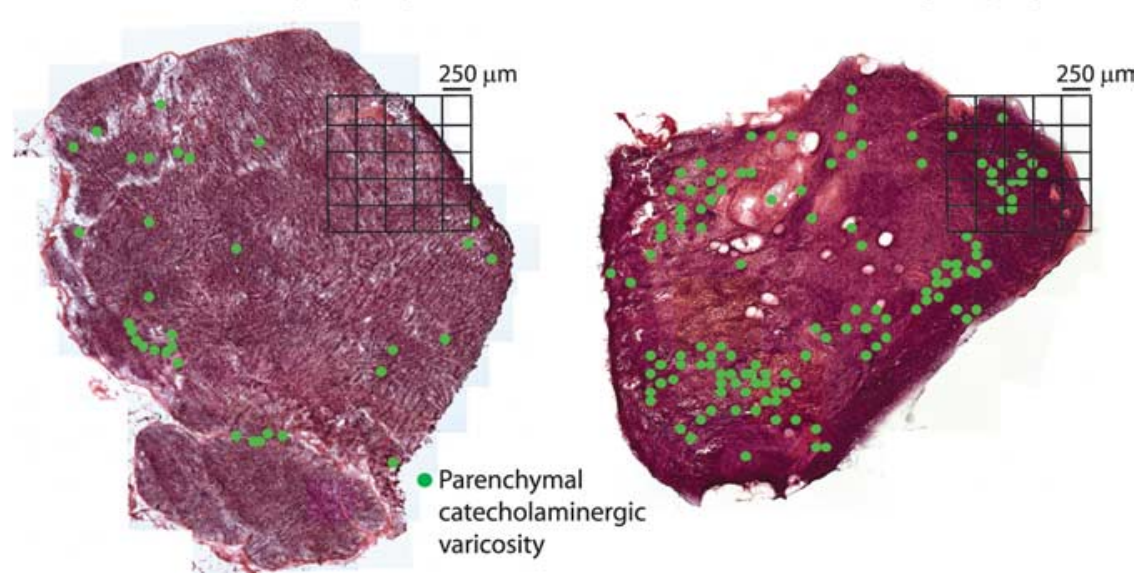

Figure 1. Social effects on lymph node innervation. $\boldsymbol{a}, \boldsymbol{b}$, Catecholaminergic neural fibers were mapped using glyoxylic acid lymph node parenchyma (a) (paracortex shown) or surrounding blood vessels (b). Scale bar, $50 \mu \mathrm{m}$. $\boldsymbol{c}$, $\boldsymbol{d}$, Innervation density was 列 asities is indicated by green dots, with density quantified relative to $250 \mu \mathrm{m}^{2}$ spatial quadrates. Across all tissues analyzed stereological statistical analyses quantified effects of social stability on parenchymal catecholaminergic varicosities and perivascular catecholaminergic varicosities. e, Error bars indicate SEM.

induce NGF transcription (e.g., via cAMP-responsive promoter elements) (McCauslin et al., 2006). Using rhesus macaque PBMCs as a model of lymphocytes that circulate through the lymph node, we exposed either unstimulated or PHA-activated PBMCs to NE concentrations comparable with those measured in secondary lymphoid organs and other solid tissues (Felten et al., 1987; Lara and Belmar, 1991; Shimizu et al., 1994). NE did not significantly alter NGF gene expression in either condition (see data in supplemental Fig. 1, available at www.jneurosci.org as supplemental material), suggesting that stress-induced NGF expression in lymphoid organs stems from other sources (Colangelo et al., 2004). Together, these results underscore the key role of NGF in structuring peripheral sympathetic innervation (LeviMontalcini, 1987; Carlson et al., 1995) and provide novel indications that its neurotrophic activity can be regulated by social processes. 
a

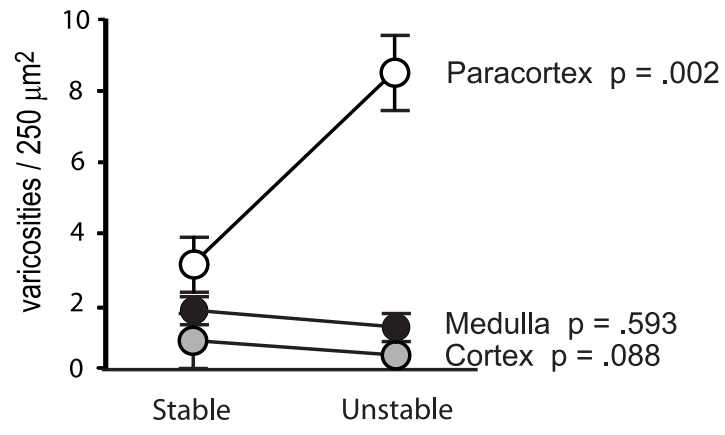

b

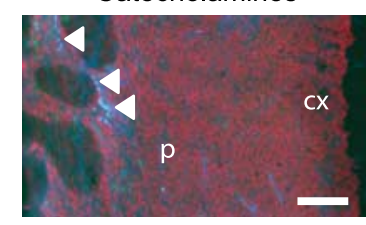

C

T lymphocytes

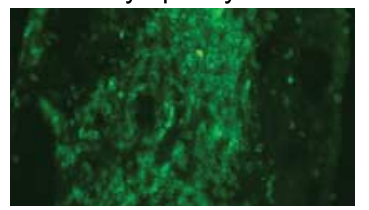

d

B lymphocytes

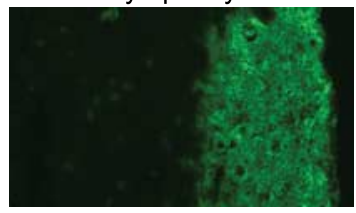

Macrophages

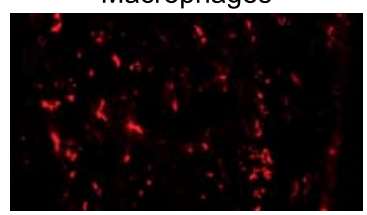

Follicular Dendritic Cells

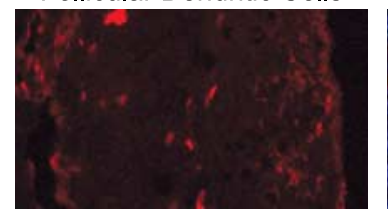

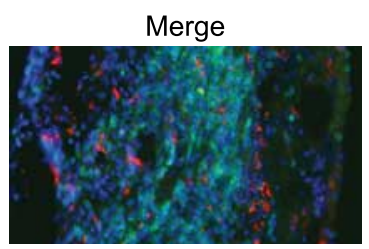

Merge

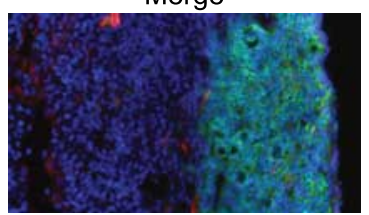

Figure 2. Impact of social conditions on lymph node anatomical subcompartments. $\boldsymbol{a}$, Density of parenchymal catecholaminergic varicosities within distinct anatomical subcompartments of lymph nodes from animals socialized under stable versus unstable conditions. Error bars indicate SEM. $\boldsymbol{b}$, Distribution of catecholaminergic varicosities (white arrowheads) in lymph node paracortex (p) and cortex (cx), shown with respect to the distribution of CD3 + T-lymphocytes (green fluorescence) and HAM56+ macrophages (red fluorescence) (c) or CD20 + B-lymphocytes (green) and follicular dendritic cells (red) (d) in adjacent lymph node sections. Nuclei are stained blue in the merged images. Scale bar, $100 \mu \mathrm{m}$.

\section{Effects of stress on lymph node function}

To assess the functional impact of stress-induced increases in lymphoid innervation density, we examined the key physiological role of the lymph node in orchestrating an immune response to infection (von Andrian and Mempel, 2003). SIV infects T-lymphocytes and macrophages, and replicates efficiently within the lymph node microenvironment (McChesney et al., 1998). To determine whether social stress impacts generalized SIV pathogenesis, we quantified plasma SIV viral load setpoint and circulating CD4+ T-lymphocyte levels in 11 rhesus macaques randomly assigned to unstable versus stable social conditions. At 36 weeks after infection with $\mathrm{SIV}_{\text {mac251 }}$, SIV plasma viral load did not differ between groups (mean \pm SE, $6.26 \times 10^{6} \pm$ $0.22 \times 10^{6}$ RNA copies $/ \mathrm{ml}$ in unstable conditions vs $6.30 \times 10^{6}$ $\pm 0.49 \times 10^{6}$ in stable conditions; difference, $\left.p=0.9239\right)$. However, socially stressed animals showed significantly lower CD4+ T-lymphocyte levels at 36 weeks after infection (mean, $255 \pm 23$ cells $/ \mathrm{mm}^{3}$ in unstable conditions vs $572 \pm 75$ in stable conditions; $p=0.0006$ ), indicating accelerated progression of SIVinduced immunopathogenesis. SIV mortality times did not differ significantly as a function of social condition (mean, $479 \pm 56 \mathrm{~d}$ after infection in unstable conditions vs $436 \pm 56$ in stable conditions; $p=0.5292$ ).
To determine the role of lymphoid tissue viral replication dynamics in stressinduced acceleration of SIV immunopathogenesis, we harvested lymph nodes from 11 rhesus macaques at 37 weeks after infection. At this time point, acute viremia had resolved to a stable viral replication setpoint, but animals did not yet show clinical illness or lymph node structural deterioration (Sloan et al., 2006). Sites of active viral replication were mapped by in situ hybridization of SIV mRNA (CantoNogues et al., 2001), and stereological statistical analysis investigated their spatial relationship to parenchymal catecholaminergic varicosities across a total of 4300 tissue quadrates (Sloan et al., 2006). Social stress increased the lymph node-wide density of SIV replication by an average of $58 \%$. Tissues from animals subject to unstable social conditions showed an average $0.264 \pm 0.015$ active viral replication sites per $250 \mu \mathrm{m}^{2}$ spatial quadrate, compared with $0.167 \pm 0.022$ from animals socialized in stable groups (difference, $p<0.0001)$.

Based on our previous data showing increased SIV replication in proximity to catecholaminergic neural fibers within the lymph node (Sloan et al., 2006), we sought to determine whether stress effects on the lymph node-wide prevalence of viral gene expression might be specifically attributable to the increased density of catecholaminergic varicosities. Stereologic analyses of in situ hybridization data showed a twofold increase in the prevalence of viral replication sites within tissue quadrates that contained one or more catecholaminergic varicosity versus none (average, $0.180 \pm 0.012$ sites per quadrate in the absence of varicosities vs $0.397 \pm 0.052$ in varicosity-containing quadrates; difference, $p<0.0001)$. However, social stress did not significantly influence the frequency of SIV replication sites within varicosity-containing tissue quadrates $(0.368 \pm 0.065$ replication sites per quadrate under stable conditions vs $0.288 \pm 0.086$ under unstable social conditions; difference, $p=0.533$ ). This suggests that stress effects on lymph node-wide SIV replication stem predominantly from the increased overall density of sympathetic varicosities (i.e., increased varicosities per lymph node), rather than from altered functional activity of individual varicosities (i.e., increased catecholamine release per varicosity). To assess this issue more directly, we performed multivariate statistical analyses at the level of individual tissue quadrates to quantify the relative contributions of structural influences (varicosity density) and functional influences (activity per varicosity) to stressinduced enhancement of SIV replication. Results from this integrative statistical model indicated a significant effect of presence versus absence of catecholaminergic varicosities $(p=0.0002)$, but did not find the quantitative magnitude of that effect to be significantly greater under unstable social conditions $(p=$ 0.664 ). Thus, social stress appears to increase SIV replication by increasing the overall prevalence of sympathetic varicosities, 


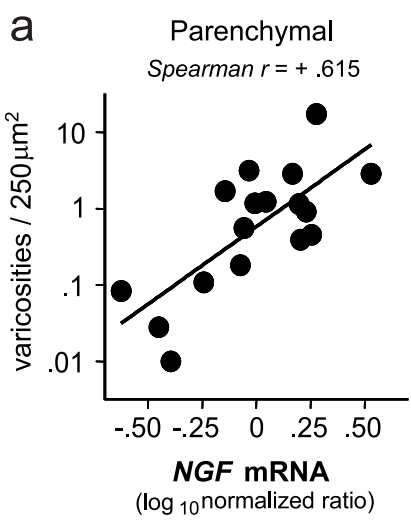

b Perivascular
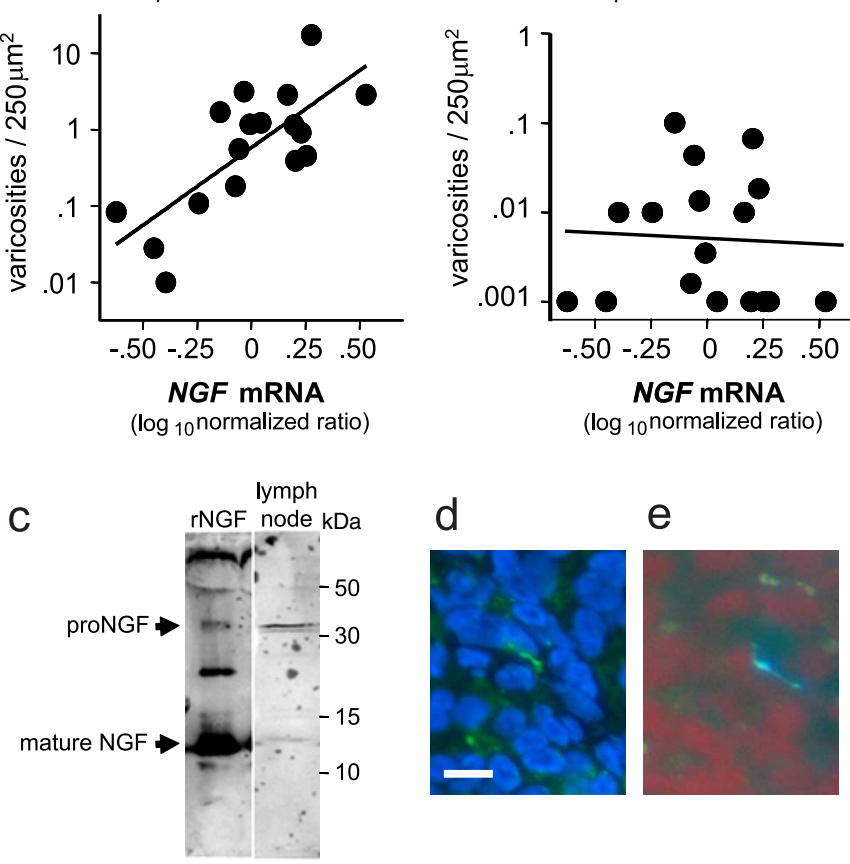

e

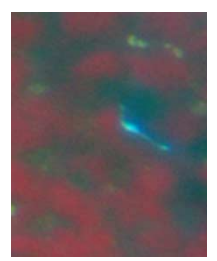

f

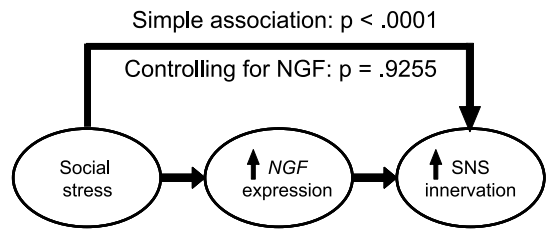

Figure 3. Social stress and NGF gene expression. $\boldsymbol{a}, \boldsymbol{b}$, NGF mRNA was quantified by real-time RT-PCR and plotted as a function of the density of parenchymal $(\boldsymbol{a})$ or perivascular $(\boldsymbol{b})$ varicosities within each lymph node. $c$, NGF protein detected by Western blot in lymph node lysate (representative of results from 3 independent experiments) detected both $13.5 \mathrm{kDa}$ mature NGF and $32 \mathrm{kDa}$ pro-NGF. $\boldsymbol{d}, \boldsymbol{e}$, Contiguous sections of a single neural fiber through adjacent lymph node sections immunostained for phosphorylated TrkA (green) with nuclei of leukocytes counterstained (blue) (d) and glyoxylic chemofluorescence for catecholamines (blue-white) with leukocytes counterstained (red) (e). Scale bar, $10 \mu \mathrm{m}$.f, Multiple regression-based mediational analysis indicated that $86 \%$ of the total effect of stress on the density of parenchymal catecholaminergic varicosities could be attributed to variations in NGF gene expression, and no significant residual effect of stress on innervation density remained after statistical control for NGF.

without significantly altering the per varicosity support for viral replication.

\section{Role of type I interferons}

Type I interferons (IFN- $\beta$ and 23 IFN- $\alpha$ family members) are well documented suppressors of HIV-1 and SIV replication, and are believed to be major physiological determinants of lentiviral replication rates (Francis et al., 1992; Agy et al., 1995; Taylor et al., 1998; Collado-Hidalgo et al., 2006). Experimental induction of IFN- $\beta$ has been shown to suppress SIV replication in vivo (Matheux et al., 2000), and our previous work has shown that NE-induced impairment of type I interferon expression represents a major mechanism by which NE enhances HIV-1 replication in vitro (Cole et al., 1998; Collado-Hidalgo et al., 2006). To determine whether stress-induced increases in SIV replication a

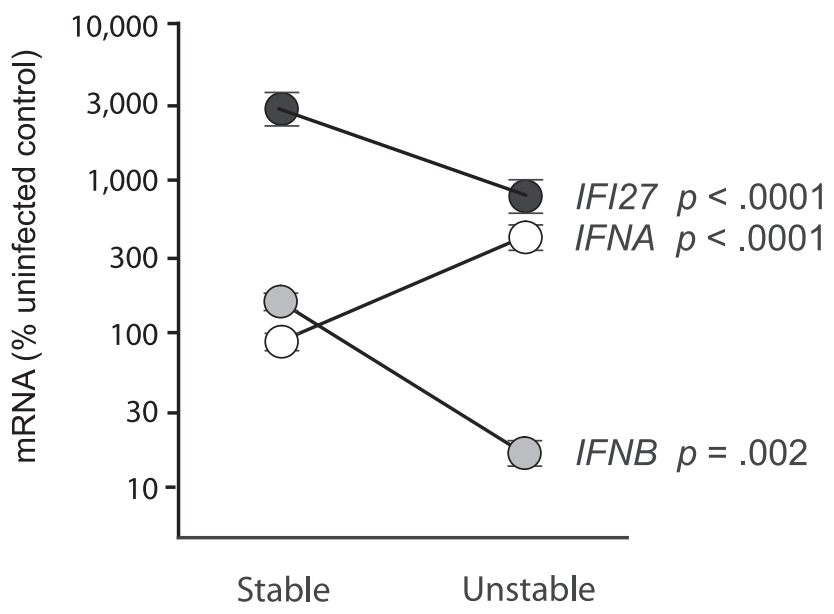

b
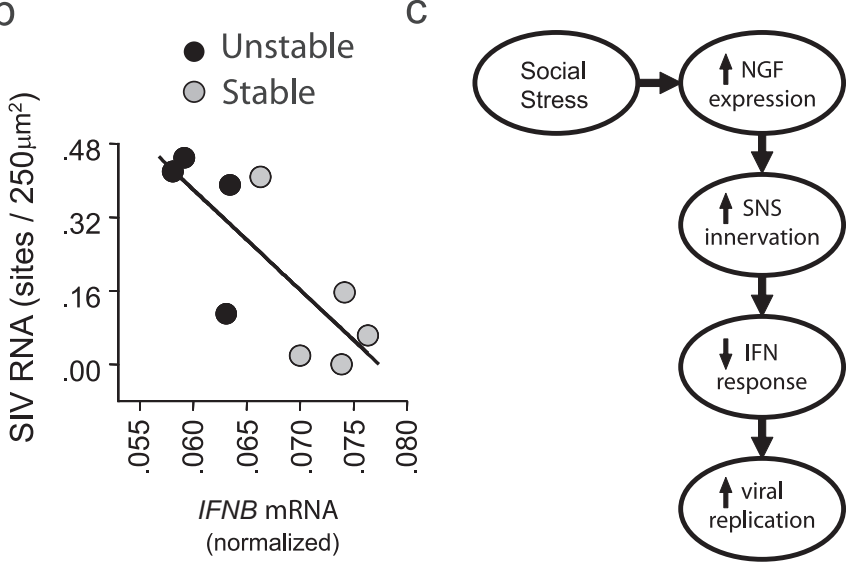

Figure 4. Type I interferons and SIV replication. $\boldsymbol{a}$, Expression of mRNA for IFNB, IFNA, and the common interferon response gene IFI27 in lymph nodes from SIV-infected animals exposed to stable versus unstable social conditions (fold change relative to uninfected control tissues). Error bars indicate SEM. $\boldsymbol{b}$, Relationship between IFNB expression and SIV replication density in lymph nodes from animals subject to stable (gray symbols) versus unstable social conditions (black symbols).c, Hypothesized model of social stress effects on NGF expression, catecholaminergic innervation, type I IFN response, and SIV replication.

within lymphoid tissue might involve the suppression of type I interferon genes, we quantified lymph node mRNA levels of IFNB and a conserved region of the 23 IFNA transcripts (Collado-Hidalgo et al., 2006). IFNB expression was inhibited by $>80 \%$ in tissues from socially stressed animals $(p=0.002)$ (Fig. $4 a$ ). In contrast, expression of IFNA family members increased by approximately fourfold in lymph nodes from stressed animals $(p<0.0001$ ) (Fig. 4a). To determine the net effect of impaired IFNB transcription and enhanced IFNA transcription, we assayed mRNA for the downstream interferon response gene IFI27, which is sensitive to all type I interferons. Results showed a substantial decrease in the overall type I interferon response, with IFI27 mRNA declining by $>60 \%$ under unstable social conditions $(p<0.0001)$ (Fig. 4a). The functional significance of this suppression was supported by analyses showing a linear relationship between increased density of SIV replication sites and decreased expression of IFNB (Fig. 4b) and IFI27 (data not shown) mRNA (both $p<0.0001$ ). Moreover, statistical mediation analyses showed that impaired type I interferon response could account for $71 \%$ of the total relationship between social stress and 
SIV gene expression across tissue samples (mediational path, $p<$ $0.0001)$. Similar results emerged from analyses of the relationship between innervation density and SIV gene expression, with type I interferon response potentially accounting for $>90 \%$ of that total relationship (mediational path, $p=0.0417$ ).

\section{Direct effects of NGF on SIV replication}

It is conceivable that stress-induced changes in SIV replication stem from a direct effect of NGF on viral replication, rather than an indirect effect of NGF that is mediated by sympathetic neural suppression of antiviral cytokines. Macaque leukocytes express mRNA for both NTRK1 (TrkA gene) and NGFR (p75NTR gene) (supplemental Fig. $2 a$, available at www.jneurosci.org as supplemental material), providing a signaling pathway for potential direct regulation of SIV replication by NGF. To assess this possibility, rhesus PBMCs were experimentally infected with SIV $_{\text {mac251 }}$ and subsequently cultured in the presence of $0.5,5$, or $50 \mathrm{ng} / \mathrm{ml}$ recombinant NGF (Lockhart et al., 1997). NGF exposure did not significantly increase SIV replication at 4 or $6 \mathrm{~d}$ after infection, as measured by relative expression of env and gag viral genes (supplemental Fig. 2b,c, available at www.jneurosci.org as supplemental material). These results are consistent with data from human clinical trials of NGF showing no effect on HIV-1 replication (McArthur et al., 2000; Schifitto et al., 2001).

\section{Discussion}

The present studies show that chronic social stress can increase the density of sympathetic innervation within the parenchymal tissue of secondary lymphoid organs from a fully mature primate. These effects are associated with increased expression of the key neurotrophic factor NGF, and do not involve changes in lymph node size, anatomical structure, or immune cell composition. Functional consequences include reduced type I interferon response to infection and a correlated increase in viral gene expression that occurs specifically in the vicinity of catecholaminergic neural fibers. Multivariate statistical analyses attribute the elevated replication of SIV specifically to the increased density of catecholaminergic varicosities within the lymph node parenchyma [as opposed to increased support for viral replication per varicosity (e.g., through enhanced neurotransmitter release)]. Effects of social stress on the structural density of lymph node innervation indicate an unanticipated degree of behaviorally induced neuroplasticity within the primate immune system, with significant implications for immunobiology and viral replication.

Analyses of neurotrophin expression implicate NGF as a likely mediator of behaviorally induced lymph node neuroplasticity, but the molecular mechanism of NGF upregulation has not been defined in the lymphoid context and is an important topic for future research. NGF can be synthesized by multiple cell types present in the lymph node, including activated T-lymphocytes and macrophages (Santambrogio et al., 1994; Frossard et al., 2004). Neuronal activity can also increase NGF gene expression (Gall and Isackson, 1989; Zafra et al., 1990; Hasan et al., 2003), raising the possibility that the stress-induced densification of lymphoid innervation might stem from antecedent increases in SNS activity. Future studies assessing the longitudinal development of stress-induced innervation and pharmacologically blocking NGF dynamics should help clarify the basis for the behaviorally induced neuroplasticity observed here.

Other physiological dynamics could potentially contribute to stress-induced upregulation of sympathetic innervation, including glucocorticoid release from the hypothalamic-pituitary-adrenal axis. Glucocorticoids have been shown to enhance sympathetic in- nervation of other tissues (e.g., ovary) (Jana et al., 2005), and they can interact with $\beta$-adrenergic signaling to enhance the transcription of NGF (Colangelo et al., 2004). However, previous studies have shown that the social stress model used here leads to a reduction in circulating glucocorticoid levels (Capitanio et al., 1998), which would not be able to explain the increased innervation observed in the current study. Stress-induced decreases in neuroinhibitory factors such as IFNG and LIF are also unlikely to explain increased innervation, because those two molecules were empirically upregulated in lymph nodes from socially stressed animals (not downregulated, as would be required to permissively increase innervation). Stress-induced upregulation of IFNG and LIF at the lymph nodewide level is unexpected, because previous studies have shown that $\beta$-adrenergic signaling generally reduces the expression of those proinflammatory mediators (Cole et al., 1998; Collado-Hidalgo et al., 2006). It is possible that these organ-level changes in IFNG and $L I F$ expression result from alterations in the recruitment of specific activated leukocyte subtypes into the lymph node (Cole et al., 1999), rather than per-cell changes in gene expression by a fixed population of leukocytes. Regardless of the molecular mechanisms involved, the empirically observed increase in lymph node IFNG and LIF levels suggest that stress-induced regulation of these molecules is not likely to mediate the observed effects of social stress on lymphoid innervation. Upregulation of NGF remains the most plausible explanation.

The present results also suggest that stress-induced alterations in lymph node innervation can significantly impact the primary physiological function of the lymph node as a mediator of immune response to infection. Stressful social conditions increased SIV replication within the lymph node and accelerated systemic immunopathogenesis, as indicated by decreased circulating CD4+ T-lymphocytes. However, plasma viral load and SIV mortality times did not differ significantly across conditions. It is unclear why the latter two indicators of disease progression failed to correlate closely with immunologically based parameters, although methodological issues may have played a role. Plasma viral load did range slightly higher under stressful conditions, but large individual variations in viral load within treatment groups ( $>10$-fold) may have undermined the statistical power to identify any existing difference. Alternatively, stress-induced changes in nonlymphoid determinants of plasma viral load (e.g., clearance rates) may have offset any stress-induced increase in viral output from lymphoid tissue. Circulating CD4+ T-lymphocyte measures were suppressed by stress, but no parallel difference in mortality times was observed. The latter finding may stem from the fact that mortality in the macaque SIV model is often driven by wasting and neurological symptoms associated with high levels of inflammation during chronic infection, rather than by the subsequent onset of opportunistic disease after immunosuppression. For the purposes of modeling human HIV-1 pathogenesis in lymphoid tissue, lymph node SIV replication and systemic CD4+ T-lymphocytes declines may be most relevant. The demonstration in this study that social stress can alter lymph node sympathetic innervation provides new insights into the molecular pathways by which biobehavioral factors can influence HIV-1 pathogenesis. Previous studies have identified several molecular mechanisms by which the sympathetic neurotransmitter norepinephrine can accelerate HIV-1 replication in vitro, including $\beta$-adrenergic upregulation of viral coreceptors (Cole et al., 1999), enhancement of viral gene transcription (Cole et al., 2001), and suppression of antiviral cytokine responses (Cole et al., 1998; Collado-Hidalgo et al., 2006). In the context of recent studies linking lymph node innervation to SIV gene expression (Sloan et al., 2006), the current data identify a novel role of sympathetic 
neuroplasticity in mediating the effects of behavioral stress on the molecular biology of viral replication in vivo.

Reductions in lymph node interferon- $\beta$ expression, concomitant with stress-induced increases in sympathetic lymphoid innervation, provide a potential mechanism for enhanced viral replication (Fig. 4c), because this type I interferon plays a key role in initiating innate antiviral responses and limiting HIV-1 replication (Francis et al., 1992; Agy et al., 1995; Taylor et al., 1998; Collado-Hidalgo et al., 2006). Indeed, recent studies suggest that $\beta$-adrenergic inhibition of IFNB response plays a central role in the ability of catecholamines to upregulate HIV-1 replication (Collado-Hidalgo et al., 2006). However, the other major type I interferon, interferon- $\alpha$, was not suppressed by stress but significantly upregulated (Fig. 4a). It is unclear why these two type I interferon species show reciprocal dynamics within the lymph node of stressed primates. The net effect of stress on total type I interferon activity appears to be suppressive, as indicated by the downregulation of the IFI27 interferon response gene, which integrates the effects of both IFNA and IFNB signaling. It is tempting to speculate that the stress-induced alterations in lymph node neural activity might potentially act to shift the relative balance of IFNA and IFNB transcription in ways that contribute to immunopathogenesis. This would be consistent with the accelerated CD4+ T-lymphocyte decline observed in stressed animals, because IFNA has been linked to HIV-induced CD4+ cell loss (Herbeuval et al., 2005; Herbeuval and Shearer, 2007).

The reciprocal regulation of IFNA and IFNB suggests a broader physiological rationale for dynamic innervation of lymphoid tissue as a pathway for CNS control of peripheral cytokine activity. The present findings are consistent with an emerging body of evidence suggesting that the autonomic nervous system plays an important physiological role in shaping the induction of immune responses by regulating T-helper cell 1 (Th1)/Th2 cytokine profiles (Cole et al., 1998; Kohm and Sanders, 1999) and helps resolve immune responses by halting production of proinflammatory cytokines (Czura and Tracey, 2005). In this context, stress-induced plasticity in the structure of lymph node innervation may provide a neurobiological mechanism by which chronic behavioral conditions can impose a long-term bias on the nature of an individual's immune response. The teleological rationale for such a bias is unclear, but the present results corroborate clinical data indicating that stable individual differences in social behavior, CNS function, and SNS activity are associated with individual differences in the risk of disease onset and pathogenesis [e.g., viral infections (Capitanio and Lerche, 1998; Cole et al., 2001; Cohen et al., 2002)].

Molecular pathways for neural-immune interaction are well defined (Lorton et al., 2001), and their functional capacity is increasingly recognized (Madden, 2001), but their physiological purpose remains unclear. The present findings suggest that it might be possible to analyze the evolution of neural-immune interactions from an ethological perspective that emphasizes the role of social behavior and lifestyle strategies in modulating individual immune responses. Close interaction with conspecifics represents a primary path for the spread of infectious disease, and it is conceivable that neural regulation of immune response could have evolved as a physiological mechanism for optimizing host resistance to infection under changing social conditions (e.g., in the presence of socially mediated threat vs support). Previous research has suggested that the quantity of social interactions can influence health outcomes (Seeman, 1996; Cohen, 2004). However, the quantity of social interaction was held constant in this study. The present data show that the specific nature of social interactions is also important and can alter the sympathetic in- nervation of lymphoid tissue in ways that may have significant implications for the pathogenesis of infectious disease.

\section{References}

Agy MB, Acker RL, Sherbert CH, Katze MG (1995) Interferon treatment inhibits virus replication in HIV-1- and SIV-infected CD4+ T-cell lines by distinct mechanisms: evidence for decreased stability and aberrant processing of HIV-1 proteins. Virology 214:379-386.

Angeletti PU, Levi-Montalcini R, Caramia F (1971) Analysis of the effects of the antiserum to the nerve growth factor in adult mice. Brain Res 27:343-355.

Bellinger DL, Lorton D, Lubahn C, Felten DL (2001) Innervation of lymphoid organs-association of nerves with cells of the immune system and their implications in disease. In: Psychoneuroimmunology, Ed 3 (Ader R, Felten DL, Cohen N, eds), pp 55-111. San Diego: Academic.

Canto-Nogues C, Jones S, Sangster R, Silvera P, Hull R, Cook R, Hall G, Walker B, Stott EJ, Hockley D, Almond N (2001) In situ hybridization and immunolabelling study of the early replication of simian immunodeficiency virus (SIVmacJ5) in vivo. J Gen Virol 82:2225-2234.

Capitanio JP, Lerche NW (1998) Social separation, housing relocation, and survival in simian AIDS: a retrospective analysis. Psychosom Med 60:235-244.

Capitanio JP, Mendoza SP, Lerche NW, Mason WA (1998) Social stress results in altered glucocorticoid regulation and shorter survival in simian acquired immune deficiency syndrome. Proc Natl Acad Sci USA 95:4714-4719.

Carlson SL, Albers KM, Beiting DJ, Parish M, Conner JM, Davis BM (1995) NGF modulates sympathetic innervation of lymphoid tissues. J Neurosci 15:5892-5899.

Chao MV, Rajagopal R, Lee FS (2006) Neurotrophin signalling in health and disease. Clin Sci (Lond) 110:167-173.

Chen Y, Dicou E, Djakiew D (1997) Characterization of nerve growth factor precursor protein expression in rat round spermatids and the trophic effects of nerve growth factor in the maintenance of Sertoli cell viability. Mol Cell Endocrinol 127:129-136.

Cohen S (2004) Social relationships and health. Am Psychol 59:676-684.

Cohen S, Hamrick N, Rodriguez MS, Feldman PJ, Rabin BS, Manuck SB (2002) Reactivity and vulnerability to stress-associated risk for upper respiratory illness. Psychosom Med 64:302-310.

Colangelo AM, Mallei A, Johnson PF, Mocchetti I (2004) Synergistic effect of dexamethasone and beta-adrenergic receptor agonists on the nerve growth factor gene transcription. Brain Res Mol Brain Res 124:97-104.

Cole SW, Korin YD, Fahey JL, Zack JA (1998) Norepinephrine accelerates HIV replication via protein kinase A-dependent effects on cytokine production. J Immunol 161:610-616.

Cole SW, Jamieson BD, Zack JA (1999) cAMP up-regulates cell surface expression of lymphocyte CXCR4: implications for chemotaxis and HIV-1 infection. J Immunol 162:1392-1400.

Cole SW, Naliboff BD, Kemeny ME, Griswold MP, Fahey JL, Zack JA (2001) Impaired response to HAART in HIV-infected individuals with high autonomic nervous system activity. Proc Natl Acad Sci USA 98:12695-12700.

Collado-Hidalgo A, Sung C, Cole S (2006) Adrenergic inhibition of innate anti-viral response: PKA blockade of type I interferon gene transcription mediates catecholamine support for HIV-1 replication. Brain Behav Immun 20:552-563.

Czura CJ, Tracey KJ (2005) Autonomic neural regulation of immunity. J Intern Med 257:156-166.

de la Torre JC, Surgeon JW (1976) Histochemical fluorescence of tissue and brain monoamines: results in 18 minutes using the sucrose-phosphateglyoxylic acid (SPG) method. Neuroscience 1:451-453.

Edwards RH, Selby MJ, Garcia PD, Rutter WJ (1988) Processing of the native nerve growth factor precursor to form biologically active nerve growth factor. J Biol Chem 263:6810-6815.

Fahnestock M, Yu G, Michalski B, Mathew S, Colquhoun A, Ross GM, Coughlin MD (2004) The nerve growth factor precursor proNGF exhibits neurotrophic activity but is less active than mature nerve growth factor. J Neurochem 89:581-592.

Farinas I (1999) Neurotrophin actions during the development of the peripheral nervous system. Microsc Res Tech 45:233-242.

Felten DL, Felten SY, Bellinger DL, Carlson SL, Ackerman KD, Madden KS, Olschowki JA, Livnat S (1987) Noradrenergic sympathetic neural interactions with the immune system: structure and function. Immunol Rev 100:225-260.

Fox CH, Tenner-Racz K, Racz P, Firpo A, Pizzo PA, Fauci AS (1991) Lym- 
phoid germinal centers are reservoirs of human immunodeficiency virus type 1 RNA. J Infect Dis 164:1051-1057.

Francis ML, Meltzer MS, Gendelman HE (1992) Interferons in the persistence, pathogenesis, and treatment of HIV infection. AIDS Res Hum Retroviruses 8:199-207.

Frossard N, Freund V, Advenier C (2004) Nerve growth factor and its receptors in asthma and inflammation. Eur J Pharmacol 500:453-465.

Gall CM, Isackson PJ (1989) Limbic seizures increase neuronal production of messenger RNA for nerve growth factor. Science 245:758-761.

Goodman LA (1960) On the exact variance of products. J Am Stat Assoc 55:708-713.

Guidry G (1999) A method for counterstaining tissues in conjunction with the glyoxylic acid condensation reaction for detection of biogenic amines. J Histochem Cytochem 47:261-264.

Guo X, Metzler-Northrup J, Lein P, Rueger D, Higgins D (1997) Leukemia inhibitory factor and ciliary neurotrophic factor regulate dendritic growth in cultures of rat sympathetic neurons. Brain Res Dev Brain Res 104:101-110.

Hasan W, Pedchenko T, Krizsan-Agbas D, Baum L, Smith PG (2003) Sympathetic neurons synthesize and secrete pro-nerve growth factor protein. J Neurobiol 57:38-53.

Hempstead BL, Martin-Zanca D, Kaplan DR, Parada LF, Chao MV (1991) High-affinity NGF binding requires coexpression of the trk protooncogene and the low-affinity NGF receptor. Nature 350:678-683.

Herbeuval JP, Shearer GM (2007) HIV-1 immunopathogenesis: how good interferon turns bad. Clin Immunol 123:121-128.

Herbeuval JP, Grivel JC, Boasso A, Hardy AW, Chougnet C, Dolan MJ, Yagita H, Lifson JD, Shearer GM (2005) CD4+ T-cell death induced by infectious and noninfectious HIV-1: role of type 1 interferon-dependent, TRAIL/DR5-mediated apoptosis. Blood 106:3524-3531.

Hoyle RH, Kenny DA (1999) Statistical power and tests of mediation. In: Statistical strategies for small sample research (Hoyle RH, ed), pp 197223. Newbury Park, CA: Sage.

Jana B, Dzienis A, Rogozinska A, Piskula M, Jedlinska-Krakowska M, Wojtkiewicz J, Majewski M (2005) Dexamethasone-induced changes in sympathetic innervation of porcine ovaries and in their steroidogenic activity. J Reprod Dev 51:715-725.

Kammer GM (1988) The adenylate cyclase-cAMP-protein kinase A pathway and regulation of the immune response. Immunol Today 9:222-229.

Kelley SP, Moynihan JA, Stevens SY, Grota LJ, Felten DL (2003) Sympathetic nerve destruction in spleen in murine AIDS. Brain Behav Immun 17:94-109.

Kim IJ, Beck HN, Lein PJ, Higgins D (2002) Interferon- $\gamma$ induces retrograde dendritic retraction and inhibits synapse formation. J Neurosci 22:4530-4539.

Klein R, Jing SQ, Nanduri V, O’Rourke E, Barbacid M (1991) The trk protooncogene encodes a receptor for nerve growth factor. Cell 65:189-197.

Kohm AP, Sanders VM (1999) Suppression of antigen-specific Th2 celldependent IgM and IgG1 production following norepinephrine depletion in vivo. J Immunol 162:5299-5308.

Lakshmanan J, Beattie GM, Hayek A, Burns C, Fisher DA (1989) Biological actions of $53 \mathrm{kDa}$ nerve growth factor as studied by a blot and culture technique. Neurosci Lett 99:263-267.

Lara HE, Belmar J (1991) Release of norepinephrine from the cat ovary: changes after ovulation. Biol Reprod 44:752-759.

Levi-Montalcini R (1987) The nerve growth factor 35 years later. Science 237:1154-1162.

Lockhart ST, Turrigiano GG, Birren SJ (1997) Nerve growth factor modulates synaptic transmission between sympathetic neurons and cardiac myocytes. J Neurosci 17:9573-9582.

Lorton D, Lubahn C, Bellinger DL (2001) Introduction to biological signaling in psychoneuroimmunology. In: Psychoneuroimmunology, Ed 3 (Ader R, Felten DL, Cohen N, eds), pp 113-160. San Diego: Academic.

Madden KS (2001) Catecholamines, sympathetic nerves and immunity. In: Psychoneuroimmunology, Ed 3 (Ader R, Felten DL, Cohen N, eds), pp 197-216. San Diego: Academic.

Madden KS, Moynihan JA, Brenner GJ, Felten SY, Felten DL, Livnat S (1994) Sympathetic nervous system modulation of the immune system. III. Alterations in $\mathrm{T}$ and $\mathrm{B}$ cell proliferation and differentiation in vitro following chemical sympathectomy. J Neuroimmunol 49:77-87.

Madden KS, Sanders VM, Felten DL (1995a) Catecholamine influences and sympathetic neural modulation of immune responsiveness. Annu Rev Pharmacol Toxicol 35:417-448.
Madden KS, Felten SY, Felten DL, Bellinger DL (1995b) Sympathetic nervous system-immune system interactions in young and old Fischer 344 rats. Ann NY Acad Sci 771:523-534.

Madden KS, Rajan S, Bellinger DL, Felten SY, Felten DL (1997) Ageassociated alterations in sympathetic neural interactions with the immune system. Dev Comp Immunol 21:479-486.

Matheux F, Lauret E, Rousseau V, Larghero J, Boson B, Vaslin B, Cheret A, De Maeyer E, Dormont D, LeGrand R (2000) Simian immunodeficiency virus resistance of macaques infused with interferon beta-engineered lymphocytes. J Gen Virol 81:2741-2750.

McArthur JC, Yiannoutsos C, Simpson DM, Adornato BT, Singer EJ, Hollander H, Marra C, Rubin M, Cohen BA, Tucker T, Navia BA, Schifitto G, Katzenstein D, Rask C, Zaborski L, Smith ME, Shriver S, Millar L, Clifford DB, Karalnik IJ (2000) A phase II trial of nerve growth factor for sensory neuropathy associated with HIV infection. AIDS Clinical Trials Group Team 291. Neurology 54:1080-1088.

McCauslin CS, Heath V, Colangelo AM, Malik R, Lee S, Mallei A, Mocchetti I, Johnson PF (2006) CAAT/enhancer-binding protein delta and cAMP-response element-binding protein mediate inducible expression of the nerve growth factor gene in the central nervous system. J Biol Chem 281:17681-17688.

McChesney MB, Sawai ET, Miller CJ (1998) Simian immunodeficiency virus. In: Persistent viral infections (Ahmed R, Chen I, eds), pp 321-345. New York: Wiley.

Miller RG (1986) Beyond ANOVA. Basics of applied statistics. New York: Wiley.

Mouton PR (2002) Principles and practices of unbiased stereology. An introduction for bioscientists. Baltimore: John Hopkins UP.

Ottaway CA, Husband AJ (1994) The influence of neuroendocrine pathways on lymphocyte migration. Immunol Today 15:511-517.

Patel TD, Jackman A, Rice FL, Kucera J, Snider WD (2000) Development of sensory neurons in the absence of NGF/TrkA signaling in vivo. Neuron 25:345-357.

Peters EM, Kuhlmei A, Tobin DJ, Muller-Rover S, Klapp BF, Arck PC (2005) Stress exposure modulates peptidergic innervation and degranulates mast cells in murine skin. Brain Behav Immun 19:252-262.

Ruit KG, Osborne PA, Schmidt RE, Johnson Jr EM, Snider WD (1990) Nerve growth factor regulates sympathetic ganglion cell morphology and survival in the adult mouse. J Neurosci 10:2412-2419.

Sanders VM, Straub RH (2002) Norepinephrine, the beta-adrenergic receptor, and immunity. Brain Behav Immun 16:290-332.

Santambrogio L, Benedetti M, Chao MV, Muzaffar R, Kulig K, Gabellini N, Hochwald G (1994) Nerve growth factor production by lymphocytes. J Immunol 153:4488-4495.

Schifitto G, Yiannoutsos C, Simpson DM, Adornato BT, Singer EJ, Hollander H, Marra CM, Rubin M, Cohen BA, Tucker T, Koralnik IJ, Katzenstein D, Haidich B, Smith ME, Shriver S, Millar L, Clifford DB, McArthur JC (2001) Long-term treatment with recombinant nerve growth factor for HIV-associated sensory neuropathy. Neurology 57:1313-1316.

Seeman TE (1996) Social ties and health: the benefits of social integration. Ann Epidemiol 6:442-451.

Shimizu N, Hori T, Nakane H (1994) An interleukin-1 beta-induced noradrenaline release in the spleen is mediated by brain corticotropinreleasing factor: an in vivo microdialysis study in conscious rats. Brain Behav Immun 8:14-23.

Sloan EK, Tarara RP, Capitanio JP, Cole SW (2006) Enhanced replication of simian immunodeficiency virus adjacent to catecholaminergic varicosities in primate lymph nodes. J Virol 80:4326-4335.

Taylor MD, Korth MJ, Katze MG (1998) Interferon treatment inhibits the replication of simian immunodeficiency virus at an early stage: evidence for a block between attachment and reverse transcription. Virology 241:156-162.

Tsui-Pierchala BA, Milbrandt J, Johnson Jr EM (2002) NGF utilizes c-Ret via a novel GFL-independent, inter-RTK signaling mechanism to maintain the trophic status of mature sympathetic neurons. Neuron 33:261-273.

von Andrian UH, Mempel TR (2003) Homing and cellular traffic in lymph nodes. Nat Rev Immunol 3:867-878.

Zafra F, Hengerer B, Leibrock J, Thoenen H, Lindholm D (1990) Activity dependent regulation of BDNF and NGF mRNAs in the rat hippocampus is mediated by non-NMDA glutamate receptors. EMBO J 9:3545-3550. 